ARTICLE

Received 2 Sep 2010 | Accepted 23 Dec 2010 | Published 1 Feb 2011

DOl: $10.1038 /$ ncomms1175

\title{
High efficiency coherent optical memory with warm rubidium vapour
}

\author{
M. Hosseini ${ }^{1,2}$, B.M. Sparkes ${ }^{1,2}$, G. Campbell',2, P.K. Lam ${ }^{1,2} \&$ B.C. Buchler ${ }^{1,2}$
}

By harnessing aspects of quantum mechanics, communication and information processing could be radically transformed. Promising forms of quantum information technology include optical quantum cryptographic systems and computing using photons for quantum logic operations. As with current information processing systems, some form of memory will be required. Quantum repeaters, which are required for long distance quantum key distribution, require quantum optical memory as do deterministic logic gates for optical quantum computing. Here, we present results from a coherent optical memory based on warm rubidium vapour and show $87 \%$ efficient recall of light pulses, the highest efficiency measured to date for any coherent optical memory suitable for quantum information applications. We also show storage and recall of up to 20 pulses from our system. These results show that simple warm atomic vapour systems have clear potential as a platform for quantum memory.

\footnotetext{
'ARC Centre of Excellence for Quantum Atom Optics, Department of Quantum Science, The Australian National University, Canberra, ACT 0200, Australia. ${ }^{2}$ ARC Centre of Excellence for Quantum Computation and Communication Technology, Department of Quantum Science, The Australian National University, Canberra, ACT 0200, Australia. Correspondence and requests for materials should be addressed to B.C.B (email: ben.buchler@anu.edu.au).
} 
A $\mathrm{n}$ ideal quantum memory must be capable of coherently storing multiple quantum states of light for on-demand recall with memory fidelity beyond the classical limit. Arriving at this goal is a challenge for experimentalists and extensive research efforts have been dedicated to the development of such a quantum memory for the last decade. The motivation for this activity is the promise of revolutionary quantum information technologies. Quantum key distribution (QKD) is already a proven technique for the secure distribution of cryptographic keys ${ }^{1,2}$, but practical implementations are limited to distances on the order of $100 \mathrm{~km}$ by the transmission losses in optical fibre or the atmosphere. Quantum computing based on optical processes ${ }^{3}$ has been shown to work in principle $e^{4}$, but this technology is limited in scale by the probabilistic nature of the optical quantum gates. A practical optical quantum memory could overcome the current limits to $\mathrm{QKD}^{5}$ and optical quantum computing ${ }^{6}$.

Many protocols have been proposed to realise such a memory device, these include electromagnetically induced transparency $(\mathrm{EIT})^{7}$, off-resonant Raman interactions ${ }^{8}$, controlled reversible inhomogeneous broadening ${ }^{9-11}$, atomic frequency combs (AFCs) ${ }^{12}$ and spin-polarization $^{13}$. Of these techniques, the most impressive efficiencies so far attained are $43 \%$ using EIT $^{14}$ and 35\% using $\mathrm{AFC}^{15}$.

In our work, we use the Gradient Echo Memory (GEM) scheme. The key to GEM is the use of an external field that induces a linear atomic frequency gradient in the direction of propagation. The frequency gradient means that the spectral components of the input light are encoded linearly along the length of the cell. Modelling has recently shown how this frequency encoding nature of GEM can be used to manipulate stored information allowing spectral compression, frequency splitting and fine dispersion control of pulses while they are stored in the memory ${ }^{16}$. Recall is achieved without $\pi$-pulses simply by reversing the field gradient (see Fig. 1a). This technique has been implemented in two-level praseodymium ions where recall

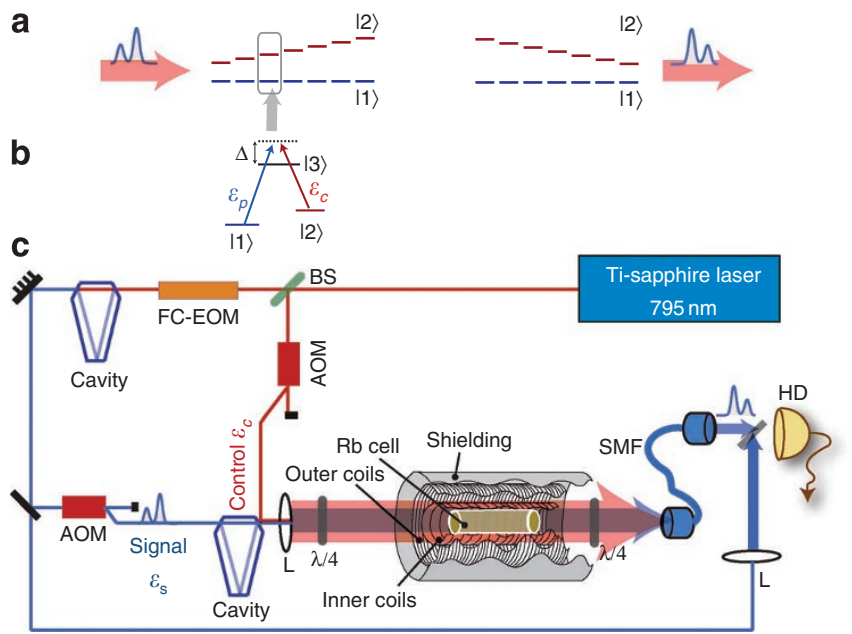

Figure 1 | Schematic view of the experiment. (a) Schematic view of the frequency gradient generated for an ensemble of two-level atoms. By switching the sign of the gradient from positive (left) to negative (right) a photon-echo is emitted in the forward direction. (b) Schematic view of $\Lambda$-atom structure, which at large detuning is equivalent to a two-level atom based on the two ground states. (c) Experimental setup showing the control (red) and signal (blue) beams with $6.8 \mathrm{GHz}$ frequency difference, collimated at radii of 6 and $3 \mathrm{~mm}$, respectively, and of identical circular polarization when they go through the cell. Heterodyne measurement is performed after the memory on the signal field. AOM, acousto-optic modulator; BS, beam splitter; FC-EOM, fibre-coupled electro-optic modulator; $\mathrm{HD}$, heterodyne detection; SMF: single-mode fibre; $\varepsilon_{s}$ and $\varepsilon_{c^{\prime}}$ signal and control field amplitudes, respectively. efficiencies of $69 \%$ have been shown ${ }^{17}$. In this solid state system, the frequency gradient is applied using an electric field to induce a Stark shift. Furthermore using AFC it has been demonstrated that optical information can be mapped into ground states of praseodymium atoms doped into $\mathrm{crystal}^{18}$. The GEM scheme has also been adapted to three-level $\Lambda$ structured atoms (Fig. 1b). This protocol makes it possible to reorder a stored pulse sequence allowing recall of any individual pulse at any chosen time ${ }^{19}$. This approach could work as an optical random-access memory for quantum information encoded in time-bin qubits. The $\Lambda$-GEM scheme uses long-lived atomic ground states for storage allowing a substantial increase of memory lifetimes compared with two-level GEM ${ }^{20,21}$. Using ground state coherences also enables a wider range of atomic systems, such as alkali atomic ensembles, which are easy to address with diode laser systems and can be contained in simple off-the-shelf vapour cells. Furthermore, it has recently been shown that vapour cells can be prepared with a single compound alkene based coating to achieve spin relaxation times of the order of few seconds ${ }^{22}$.

Here, we present results of a $\Lambda$-GEM experiment in which memory efficiencies $>80 \%$ have been coherently measured from a warm rubidium vapour. We also report on the storage and recall of 20 pulses. The current results show great potential of this memory scheme for storage of quantum states of light.

\section{Results}

Signal and control beams. The experimental setup is shown in Figure 1c. To prepare the signal beam, a fibre coupled phase-modulator was used to produce sidebands at the ground-state hyperfine splitting of ${ }^{87} \mathrm{Rb}(6.8 \mathrm{GHz})$. The beam was sent to a cavity (finesse $=100$ ) resonant with the $+6.8 \mathrm{GHz}$ sideband to filter out the carrier and the $-6.8 \mathrm{GHz}$ sideband. Both the coupling and signal beams were intensity controlled using acousto-optic modulators. Signal pulses were prepared with a peak power of $<2 \mathrm{~mW}$. The signal pulses and control beam were then combined with the same linear polarization using another cavity (also finesse $=100$ ) resonant with the signal field. The control and signal fields were converted to circular polarization and sent into the gas cell. After the cell, the signal field was coupled to a single mode fibre and sent to a heterodyne detection system. The coupling of the control field to this fibre was weak because of its larger mode diameter (for more details see Methods section).

Figure 2a shows the Raman absorption line as a function of twophoton detuning (detuning from the Raman resonance) (i) with and (ii) without the applied magnetic field gradient. The absorption is sensitive to alignment, which was optimized for for the broadened feature. With the applied broadening the absorption is $\sim 99 \%$. This limits the maximum possible recall efficiency of our memory ${ }^{23}$ to $0.99^{2}=98 \%$.

Pulse storage and recall. The results of storage and recall experiments are shown in Figure $2 b$. The input pulse, shown in black, has a $1 / \mathrm{e}^{2}$ width of $2 \mu \mathrm{s}$. We measure the power in this input pulse by recording the far off-resonance transmission through the gas cell without the control field. We measured no noticeable absorption from the atomic ensemble under these conditions and we can use the total energy in this pulse (the area under the curve) to normalize the recall efficiency of our storage experiments.

In Figure $2 \mathrm{~b}$ after flipping the magnetic field gradient, we recall the signal light with a maximum efficiency of $87 \%$. The storage time in this case is $3.7 \mu$ s peak-to-peak, or exactly one pulse width between the $1 / \mathrm{e}^{2}$ points power levels to ensure complete separation of the input and recalled pulses. The recall efficiency drops rapidly for longer storage times, although for these data the control field was on at all times. In Figure 2c, we show results of recall experiments, in which the control field was switched off during the storage phase of the experiment. In this case, we find slower decay of the recalled pulse, as we have now reduced the decoherence caused by the 

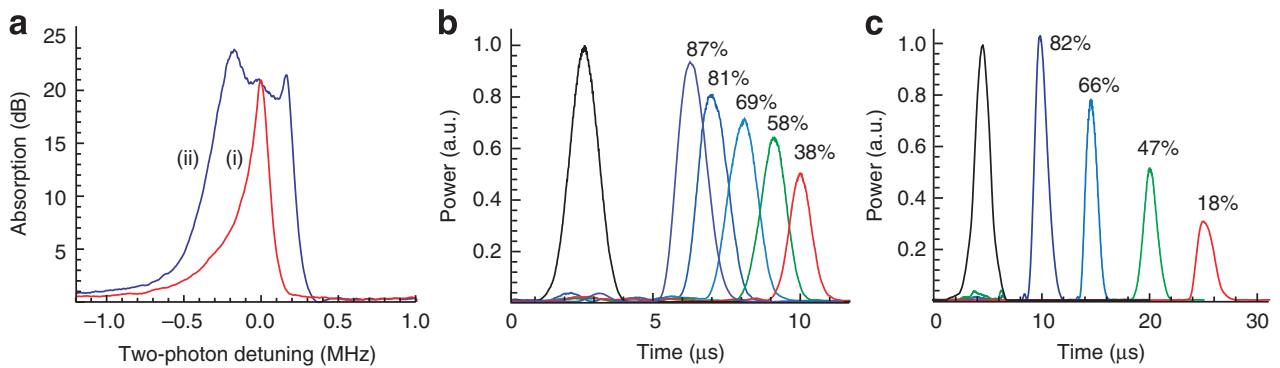

Figure 2 | Raman absorption line and input-echo pulses. (a) (i) The Raman absorption line before broadening. (ii) The Raman absorption line after application of the magnetic field gradient. This was observed with a single frequency continuous wave signal beam, whereas the frequency of the control beam was scanned. (b) Storage and recall data with an input pulse duration of $2 \mu \mathrm{s}$. (c) Storage and recall data with an input pulse duration of $3 \mu$ s and the control field is turned off during the storage time to reduce the decay rate of the storage. For both $\mathbf{b}$ and $\mathbf{c}$ the far off-resonant transmitted input pulse, which is used to normalize our recall efficiency, is shown in black. The control field power was $370 \mathrm{~mW}$.
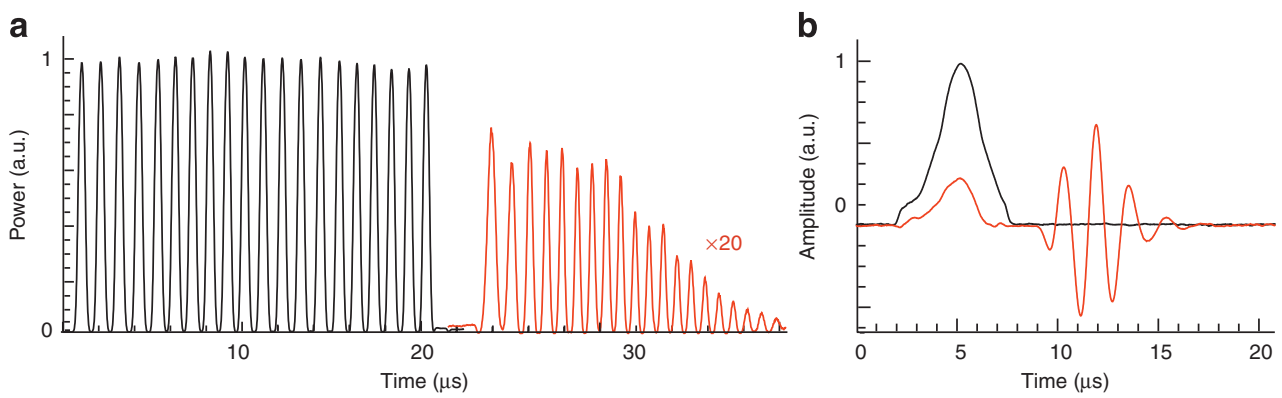

Figure 3 | Results of 20 pulse storage and frequency shifting. (a) Amplitude of 20 Gaussian input (black) and echo pulses (red) with total recall efficiency of $2 \%$. (b) The input pulse (black) is stored and recalled with an introduced offset to the magnetic field gradient. The red curve denotes the straight transmitted pulse and the recall pulse. The interference pattern shows that the pulse is coherent with the original light field and is shifted by $600 \mathrm{kHz}$.

control field. The pulses in these data are slightly compressed on recall due to a higher magnetic field gradient used to recall the signal light, which is the reason for the peak recalled power exceeding the input peak power. We can achieve slightly higher efficiency using compressed pulses as the total storage time in the medium is reduced.

In Figure 3a, we show the storage and recall of 20 Gaussian pulses with an overall efficiency of $2 \%$. For this experiment, the control field power was reduced from 370 to $64 \mathrm{~mW}$ to reduce the decay rate of the memory. The lower optical depth in this case limits the efficiency of the storage and recall. From this data we can infer a delaybandwidth product ${ }^{24}$ of $\sim 40$ for the our memory. This is comparable to a recent demonstration of 64 pulses delayed using AFC with efficiency of $1.3 \%$ (ref. 25). Figure $3 \mathrm{~b}$ demonstrates frequency shifted recall of a pulse ${ }^{16}$. This was achieved by applying an offset magnetic field after the magnetic field gradient was flipped to increase the splitting of the atomic ground states. On recall, the pulse is shifted by the added splitting, which in this case is $600 \mathrm{kHz}$, as seen by the interference fringes in the heterodyne signal. The presence of interference fringes proves the coherent recall of our memory.

\section{Discussion}

To understand the limits of our atomic system, we now examine some theoretical aspects of the scheme. Assuming far-detuned Raman interaction, the system depicted in Figure 1a can be treated as a quasi two-level atom. One can write the Maxwell-Bloch equations for the atom-light interaction scheme depicted in Figure 1a in terms of atomic coherence $\sigma_{12}$ and electric field amplitude $\varepsilon_{\mathrm{s}}$,

$$
\begin{aligned}
& \dot{\hat{\sigma}}_{12}=-\left(\gamma_{12}+i \delta(z, t)\right) \hat{\sigma}_{12}-i g \frac{\Omega_{c}}{\Delta} \hat{\varepsilon}_{s} \\
& \frac{\partial}{\partial z} \hat{\varepsilon}_{s}=i \mathcal{N} \frac{\Omega_{c}}{\Delta} \hat{\sigma}_{12},
\end{aligned}
$$

where $\mathcal{N}=g N / c$ is the effective linear atomic density, $g$ is the atomlight coupling, $\delta(z, t)$ is the additional splitting between the two ground states (two-photon detuning) due to the magnetic gradient, $\Omega_{c}$ is the control field Rabi frequency, and $\Delta$ is detuning from the excited state. The dephasing rate of $|1\rangle$ to $|2\rangle, \gamma_{12}$ in equation (1), includes population shuffling due to atomic collision and other dephasing mechanisms such as the power broadening ${ }^{26}$ caused by spontaneous Raman scattering. On the basis of our experimental conditions, the rate of spontaneous emission due to the signal beam is found to be $2 \pi \times 1 \mathrm{~Hz}$ at pulse peak intensity. The scattering rate due to the control field, on the other hand, is found to be $\sim 2 \pi \times 30 \mathrm{kHz}$ at $\Delta=3 \mathrm{GHz}$ and a power of $370 \mathrm{~mW}$.

In the case of EIT systems, the effect of four-wave mixing attributed to the interaction of the control field with the $F=1, m_{F}=1$ level is proven to affect memory performance at large optical depths ${ }^{14,27}$. In these experiments, a double- $\Lambda$ transition appeared in the system aided by the presence of a seed stokes field at $-6.8 \mathrm{GHz}$ generated by the EOM. In our experiment, the $-6.8 \mathrm{GHz}$ sideband seed is rejected by two cavities that are on resonance only with the $+6.8 \mathrm{GHz}$ sideband, thereby eliminating any contribution of coherent four-wave mixing to our system.

To understand the contribution of the control field-induced scattering, we investigated memory behaviour both in the presence and absence of the control field during the storage time. The control field can be switched off during the storage time without affecting the memory protocol. We can gain some insight into our system by considering a simple model that includes an atomic diffusion time, $\tau_{\mathrm{d}}$, a total ground state decoherence rate, $\tau_{0}$, and a maximum possible memory efficiency, $\eta_{0}$, limited by the optical depth. The efficiency $\eta_{\mathrm{m}}$ will then be given by

$$
\eta_{\mathrm{m}}=\eta_{0} \mathrm{e}^{-\left(t / \tau_{\mathrm{d}}\right)^{2}} \mathrm{e}^{-t / \tau_{0}}
$$



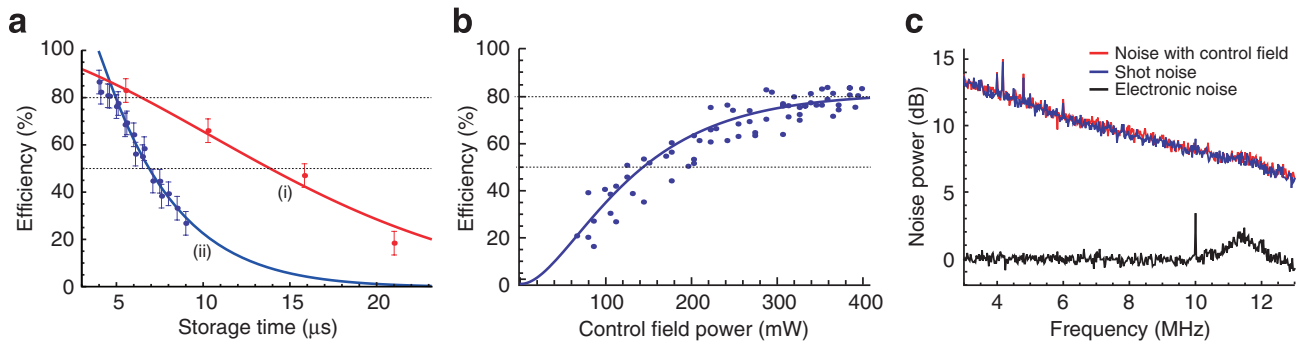

Figure 4 | Control field effect on efficiency and noise. (a) Echo efficiency as a function of storage time. (i, red) Data taken for $3 \mu$ s pulse while the control field with power of $370 \mathrm{~mW}$ was switched off during the storage time. (ii, blue) Data taken for $2 \mu \mathrm{s}$ pulse while the control field with power of $290 \mathrm{~mW}$ was kept on during the storage time. Error bars indicate the detection error derived from fluctuation of the amplitudes of pulses. (b) Efficiency of photon echoes of a $3 \mu$ s pulse as a function of control field power. The solid line is the theoretical predictions taking into account diffusion time of $22 \mu \mathrm{s}$, control field-induced scattering and ground state decoherence rate of $2 \pi \times 3.5 \mathrm{kHz}$. (c) Variance of the probe field mode measured using heterodyne detection. Curves represent electronic noise (black), shot noise (blue) and noise with the control field switched on (red). Measurements were made with a resolution bandwidth $=3 \mathrm{kHz}$, video bandwidth $=30 \mathrm{kHz}$ and five averages. The control beam was filtered out of this measurement using and additional gas cell containing warm ${ }^{85} \mathrm{Rb}$

Figure $4 \mathrm{a}(\mathrm{i})$ shows the efficiency as a function of storage time when the control field is on. Taking into account the signal beam radius of $3 \mathrm{~mm}$ and 0.5 Torr $\mathrm{Kr}$ buffer gas, one can calculate the diffusion time of the atoms, defined as the time that a fraction $1 / \mathrm{e}^{2}$ of atoms have moved a distance greater than the radius of the signal beam, to be $\tau_{\mathrm{d}}=22 \mu \mathrm{s}$. This value was fixed in our model allowing us to fit only the ground state decay time, which was determined to be $\tau_{0}=4 \mu \mathrm{s}$ corresponding to a decay rate of $2 \pi \times 40 \mathrm{kHz}$. This is consistent with the scattering rate of $2 \pi \times 30 \mathrm{kHz}$ calculated above, from which we conclude that our system is limited in this regime by control-beaminduced scattering.

We note, however, that this may not be the case for very short storage times where the decay is not exponential and does not agree with our simple model. This effect can be attributed to the highly photonic nature of the memory for short storage times. The pulse, in this case, has yet to be fully mapped into the atomic spin wave. The impact of the scattering, collisional and diffusion decay terms will vary as the light is absorbed into the atomic ensemble leading to decay of the memory that differs from the model at short times.

The scattering rate can be minimized by switching off the control field during the storage. Atomic diffusion then becomes the dominant decay mechanism, hence, a more Gaussian-like decay is expected. The curve in Figure $4 \mathrm{a}(\mathrm{i})$ is the result of a convolution of a Gaussian decay function $\left(\tau_{\mathrm{d}}=22 \mu \mathrm{s}\right)$ because of diffusion and an exponential decay function due to the ground state decoherence. The fitted exponential decay time in this case is $\tau_{0}=60 \mu \mathrm{s}$, giving a decay rate of $2 \pi \times 2.6 \mathrm{kHz}$. This is still much higher than the collisionlimited ground state dephasing time, but this is expected because the decay rate in this case varies as the control beam is switched on and off over the course of the experiment.

We also studied the effect of the control field power on the memory efficiency for storage times of one-pulse width. As can be seen in Figure $4 \mathrm{~b}$, the recall efficiency saturates with increasing control field power. This is because increasing $\Omega_{c}$ can effectively provide higher optical depth as long as $\Delta \gg \Omega_{c}$. In this case, the effective atomlight coupling strength can be described as $g^{\prime}=g \Omega_{c} / \Delta$. If $\Omega_{c}$ is further increased beyond this limit, the system can no longer be described by a simple quasi two-level atomic ensemble and there is no further improvement in optical depth.

The presence of the strong control field could lead to noise sources in our memory due to spontaneous emission and Raman scattering into the mode of the probe beam. To investigate these possible noise sources we measured the noise spectrum of the probe mode as shown in Figure 4c. With no control field present, we observe the shot noise of our detection system (blue), which lies $10 \mathrm{~dB}$ above the electronic noise floor (black). With the control field switched

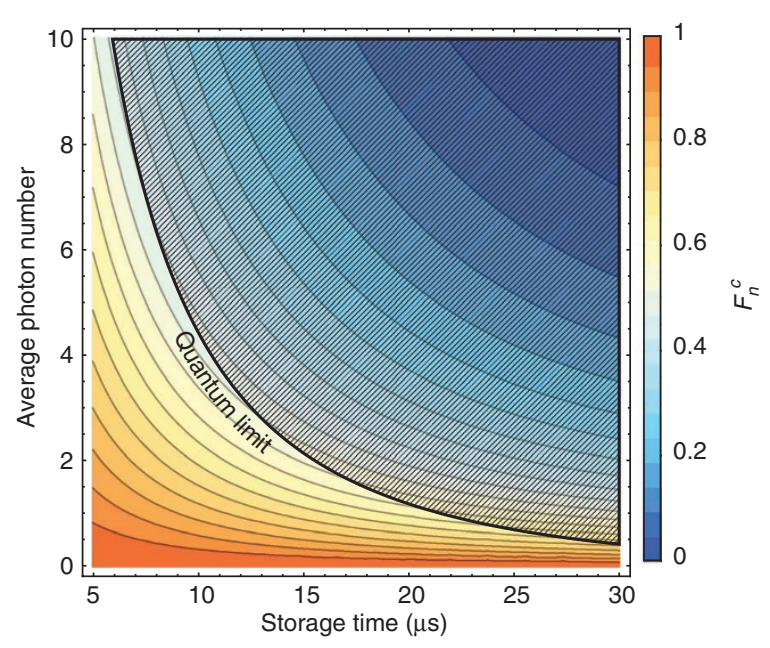

Figure 5 | Implied quantum memory performance for coherent state storage. The quantum limit is calculated assuming the efficiency fitted to experimental data in Figure $4 \mathrm{a}(\mathrm{i})$ and equation (3).

on (red), we observe no change in the noise level recorded by our heterodyne system. If there where photons added to the mode of the probe field, then we would see added noise around $8 \mathrm{MHz}$, which is the frequency offset of the heterodyne beam from the probe frequency. The absence of extra noise at this frequency is strong evidence that our memory is not prone to noise sources that could impact on quantum state storage. The data shown in Figure $4 b, c$, along with the coherent detection, is the strong evidence that our high efficiency recall is not due to any nonlinear process or incoherent contamination of the signal mode by the strong control beam.

The best way to quantify the efficacy of a quantum memory will depend on the application. To quantify how the measured efficiency of our memory would translate into a coherent state quantum memory, we can follow the model presented by He et $a .^{28}$, in which it is shown that a linear quantum memory has fidelity $\left(F_{n}^{c}\right)$

$$
F_{n}^{c}>\frac{1}{1+\bar{n}\left(1-\sqrt{\eta_{\mathrm{m}}}\right)^{2}}
$$

for coherent states with average photon number $\bar{n}$ and memory efficiency $\eta_{\mathrm{m}}$. Given that our memory is linear, and assuming that no extra noise is added to the stored states, we can calculate the 
range of coherent amplitudes for which it can fuction as a quantum memory, as shown in Figure 5. This shows, for example that we could store coherent states up to $\bar{n}=10$ for times $<6 \mu \mathrm{s}$, or states with $\bar{n}=1$ for $21 \mu \mathrm{s}$.

In conclusion, we have shown light storage in warm vapour gas with $>80 \%$ memory efficiency using $\Lambda$-GEM technique. All measurements were made using coherent heterodyne detection. We have demonstrated a time-bandwidth product of $\sim 40$ and controlled frequency shift of the recalled pulse. The decay rate of our memory can be controlled by minimizing the use of the control beam to the extent that for longer storage times we become limited by atomic diffusion. If applied to coherent state storage, our scheme could perform as a quantum memory for states containing up to 10 photons.

\section{Methods}

Experiment. Our atomic ensemble consists of a cylindrical cell (length and diameter of $20 \mathrm{~cm}$ and $25 \mathrm{~mm}$, respectively) containing ${ }^{87} \mathrm{Rb}$ atoms mixed with 0.5 Torr of $\mathrm{Kr}$ buffer gas, used to increase the time-of-flight of atoms inside the beams. The time-of-flight of atoms inside the signal and the control beam is calculated to be 45 and $180 \mu$ s, respectively. We consider the off-resonant Raman interaction of a weak signal field and a strong control field, both having $\sigma^{+}$polarization, addressing $|1\rangle$ to $|3\rangle\left(S_{1 / 2}, F=1 \rightarrow P_{1 / 2}, F^{\prime}=2\right)$ and $|2\rangle$ to $|3\rangle\left(S_{1 / 2}, F=2 \rightarrow P_{1 / 2}\right.$, $\left.F^{\prime}=2\right)$ transitions, respectively, as shown in Figure $1 b$. These fields are blue-detuned by $\Delta=3 \mathrm{GHz}$ from the excited state.

The temperature of the cell was held around $78^{\circ} \mathrm{C}$ using a bifilar resistive heater wrapped around the cell. We estimate the number of atoms in the signal beam mode volume to be $\simeq 4 \times 10^{12}$. Considering the corresponding Clebsch-Gordan coefficients ${ }^{29}, \sim 65 \%$ of these atoms will be located in $F=1, m_{F}=1$ state with the rest of the population located in $F=2, m_{F}=2$. To create the frequency gradient, two specially wound magnetic coils surrounding the cell were used to produce opposing linearly varying Zeeman shifts along the cell ${ }^{19}$. Switching current between these coils created a switchable atomic frequency gradient in our storage medium. To recall the pulse, one coil is switched off and one is switched on within 0.5 and $2.5 \mu \mathrm{s}$, respectively. The DC magnetic field is normally set to $6 \mathrm{G}$ and the typical value of the gradient field is $20 \mathrm{mG} \mathrm{cm}^{-1}$. The cell and coils are surrounded with $\mu$-metal to reduce the influence of the Earth's magnetic field.

\section{References}

1. Gisin, N., Ribordy, G., Tittel, W. \& Zbinden, H. Quantum cryptography. Rev. Mod. Phys. 74, 145-195 (2002).

2. Gisin, N. \& Thew, R. Quantum communication. Nat. Photonics 1, 165-171 (2007).

3. Knill, E., Laflamme, R. \& Milburn, G. A scheme for efficient quantum computation with linear optics. Nature 409, 46-52 (2001).

4. Politi, A., Matthews, J. C. F. \& O'Brien, J. L. Shor's quantum factoring algorithm on a photonic chip. Science 325, 1221 (2009).

5. Duan, L.- M., Lukin, M., Cirac, J. I. \& Zoller, P. Long-distance quantum communication with atomic ensembles and linear optics. Nature 414, 413-418 (2001).

6. Kok, P. et al. Linear optical quantum computing with photonic qubits. Rev. Mod. Phys. 79, 135-174 (2007).

7. Fleischhauer, M. \& Lukin, M. Dark-state polaritons in electromagnetically induced transparency. Phys. Rev. Lett. 84, 5094-5097 (2000).

8. Reim, K. F. et al. Towards high-speed optical quantum memories. Nat. Photonics 4, 218-221 (2010).

9. Moiseev, S. \& Kroll, S. Complete reconstruction of the quantum state of a single-photon wave packet absorbed by a doppler-broadened transition. Phys. Rev. Lett. 87, 173601 (2001).

10. Moiseev, S. A., Tarasov, V. F. \& Ham, B. S. Quantum memory photon echo-like techniques in solids. J. Optics B Quantum and Semiclassical Opt. 5, S497-S502 (2003).

11. Nilsson, M. \& Kroll, S. Solid state quantum memory using complete absorption and re-emission of photons by tailored and externally controlled inhomogeneous absorption profiles. Opt. Commun. 247, 393-403 (2005).
12. Afzelius, M., Simon, C., de Riedmatten, H. \& Gisin, N. Multimode quantum memory based on atomic frequency combs. Phys. Rev. A 79, 052329 (2009).

13. Julsgaard, B., Sherson, J., Cirac, J., Fiurasek, J. \& Polzik, E. Experimental demonstration of quantum memory for light. Nature 432, 482-486 (2004).

14. Phillips, N. B., Gorshkov, A. V. \& Novikova, I. Optimal light storage in atomic vapor. Phys. Rev. A 78, 023801 (2008).

15. Amari, A. et al. Towards an efficient atomic frequency comb quantum memory. J. Lumin. 130, 1579-1585 (2010).

16. Buchler, B. C., Hosseini, M., Hetet, G., Sparkes, B. M. \& Lam, P. K. Precision spectral manipulation of optical pulses using a coherent photon echo memory. Opt. Lett. 35, 1091-1093 (2010).

17. Hedges, M., Longdell, J., Li, Y. \& Sellars, M. Efficient quantum memory for light. Nature 465, 1052-1056 (2010).

18. Afzelius, M. et al. Demonstration of atomic frequency comb memory for light with spin-wave storage. Phys. Rev. Lett. 104, 040503 (2010).

19. Hosseini, M. et al. Coherent optical pulse sequencer for quantum applications. Nature 461, 241-245 (2009).

20. Hetet, G. et al. Photon echoes generated by reversing magnetic field gradients in a rubidium vapor. Opt. Lett. 33, 2323-2325 (2008).

21. Hetet, G., Longdell, J. J., Sellars, M. J., Lam, P. K. \& Buchler, B. C. Multimodal properties and dynamics of gradient echo quantum memory. Phys. Rev. Lett. 101, 203601 (2008)

22. Balabas, M. V. et al. High quality anti-relaxation coating material for alkali atom vapor cells. Opt. Express 18, 5825-5830 (2010).

23. Longdell, J. J., Hetet, G., Lam, P. K. \& Sellars, M. J. Analytic treatment of controlled reversible inhomogeneous broadening quantum memories for light using two-level atoms. Phys. Rev. A 78, 032337 (2008).

24. Tidstrom, J., Janes, P. \& Andersson, L. Delay-bandwidth product of electromagnetically induced transparency media. Phys. Rev. A 75, 053803 (2007).

25. Usmani, I., Afzelius, M., de Riedmatten, H. \& Gisin, N. Mapping multiple photonic qubits into and out of one solid-state atomic ensemble. Nat. Commun $1,12(2010)$.

26. Citron, M., Gray, H., Gabel, C. \& Stroud, C. Experimental study of power broadening in a two-level atom. Phys. Rev. A 16, 1507-1512 (1977).

27. Phillips, N., Gorshkov, A. \& Novikova, I. Slow light propagation and amplification via electromagnetically induced transparency and four-wave mixing in an optically dense atomic vapor. J. Mod. Opt. 56, 1916-1925 (2009).

28. He, Q., Reid, M., Giacobino, E., Cviklinski, J. \& Drummond, P. Dynamical oscillator-cavity model for quantum memories. Phys. Rev. A 79, 022310 (2009).

29. Steck, D. A. Rubidium 87 d line data, http://steck.us/alkalidata/ (2009).

\section{Acknowledgments}

We thank M. Sellars for enlightening discussions. This work was supported by the Australian Research Council through the Centre of Excellence for Quantum Atom Optics (CE0348178) and the Centre of Excellence for Quantum Computation and Communication Technology (CE11E0096).

\section{Author contributions}

Experiments, measurements and data analysis were performed by M.H. with assistance of B.M.S. and G.C. for data collection and experimental preparation. The project was planned and supervised by B.C.B. and P.K.L. The manuscript was written by B.C.B. and M.H. with the assistance of all other authors.

\section{Additional information}

Competing financial interests: The authors declare no competing financial interests.

Reprints and permission information is available online at http://npg.nature.com/ reprintsandpermissions/

How to cite this article: Hosseini, M. et al. High efficiency coherent optical memory with warm rubidium vapour. Nat. Commun. 2:174 doi: 10.1038/ncomms1175 (2011)

License: This work is licensed under a Creative Commons Attribution-NonCommercialNoDerivative Works 3.0 Unported License. To view a copy of this license, visit http:// creativecommons.org/licenses/by-nc-nd/3.0/ 